\title{
Lyapunov Stability of the Generalized Stochastic Pantograph Equation
}

\author{
Ramazan Kadiev ${ }^{1}$ and Arcady Ponosov (D) $^{2}$ \\ ${ }^{1}$ Dagestan Research Center of the Russian Academy of Sciences and Department of Mathematics, Dagestan State University, \\ Makhachkala 367005, Russia \\ ${ }^{2}$ Norwegian University of Life Sciences, Faculty of Sciences and Technology, P.O. Box 5003, N-1432 Ås, Norway
}

Correspondence should be addressed to Arcady Ponosov; arkadi@nmbu.no

Received 31 January 2018; Accepted 14 May 2018; Published 19 June 2018

Academic Editor: Qamar Din

Copyright (C) 2018 Ramazan Kadiev and Arcady Ponosov. This is an open access article distributed under the Creative Commons Attribution License, which permits unrestricted use, distribution, and reproduction in any medium, provided the original work is properly cited.

The purpose of the paper is to study stability properties of the generalized stochastic pantograph equation, the main feature of which is the presence of unbounded delay functions. This makes the stability analysis rather different from the classical one. Our approach consists in linking different kinds of stochastic Lyapunov stability to specially chosen functional spaces. To prove stability, we check that the solutions of the equation belong to a suitable space of stochastic processes, instead of searching for an appropriate Lyapunov functional. This gives us possibilities to study moment stability, stability with probability 1, and many other stability properties in an efficient way. We show by examples how this approach works in practice, putting emphasis on delay-independent stability conditions for the generalized stochastic pantograph equation. The framework can be applied to any stochastic functional differential equation with finite dimensional initial conditions.

\section{Introduction}

In this paper we study Lyapunov stability of the stochastic pantograph equation (see, e.g., [1-3]):

$$
\begin{aligned}
d x(t)= & {\left[A x(t)+\sum_{j=0}^{m_{0}} A_{j 0} x\left(g_{j 0} t\right)\right] d t } \\
& +\sum_{l=1}^{m-1} \sum_{j=0}^{m_{l}} A_{j l} x\left(g_{j l} t\right) d \mathscr{B}_{l}(t),
\end{aligned}
$$

where $0<g_{j l}<1$, and its generalizations (see (21) in Section 4). A very good and comprehensive description of the role of the classical pantograph equation and its stochastic counterpart, including historical comments, can be found in the paper [2]. Let us only mention that generalizations of the pantograph equations have also attracted attention of many mathematicians; see, e.g., [4-11] and the references therein.

Stability analysis of (1) and (21) has a special feature: the delay is unbounded, so that many methods, including those based on Lyapunov-Krasovskii functionals, are inapplicable. One uses therefore various special techniques, which can, e.g., be found in the papers $[12,13]$ (the stochastic case) and [5] (the deterministic case). These techniques help to produce verifiable stability criteria, mostly in the case of the classic pantograph equation (1).

Our approach goes back to the framework developed in the monographs [14] (for linear differential equations in Banach spaces) and [15] (for linear deterministic functional differential equations), where Lyapunov stability is replaced by input-to-state stability, i.e., the property of the equation where its solutions belong to certain linear topological spaces and continuously depend (in the corresponding topology) on the initial data. In the stochastic case this approach is outlined in [16]. On the other hand, (1) and (21) possess a very specific property: their initial conditions are finite dimensional, i.e., identical to the ones for ordinary differential equations. This considerably simplifies the analysis of the input-tostate stability, as all linear finite dimensional operators are bounded, and we only need to prove that all solutions of the 
equation belong to a certain topological space. For brevity, we will call this property $M$-stability keeping in mind that this is, in fact, a particular case of the input-to-state stability for linear equations with finite dimensional spaces of initial data.

The idea of how to verify the property of input-to-state stability for linear deterministic functional differential equations goes back to the papers of N.V.Azbelev and his students (see [15] and the references therein) who call their technique the $W$-method. It is somewhat similar to Lyapunov's direct method. But instead of seeking a Lyapunov function(al) one aims to find a suitable reference equation which possesses the prescribed asymptotic property and which then is used to regularize the original equation. Like Lyapunov's method, the $W$-method also provides necessary and sufficient stability conditions. The $W$-method proven to be rather efficient for many classes of delay equations, especially those where searching for Lyapunov functionals seems to be difficult. Equations with infinite delays can serve as a prominent example of such a class.

In [17], the method was for the first time applied to linear stochastic functional differential equations and developed further by the authors in the series of publications (see the review article [16]). The first efficient stability conditions for stochastic differential equations with unbounded delays, obtained by the $W$-method, were presented in the paper [7]. In the present paper we develop this approach further by concentrating on specific stochastic equations with unbounded delays and finite dimensional initial conditions: the pantograph equation (1) and its generalization (21). In the examples below (see Section 4) we only use the simplest reference equation $\dot{x}+\alpha x=g$, where $\alpha>0$ is a parameter, variation of which ensures best possible stability conditions. More sophisticated reference equations (e.g., those including delays) can be found in other publications of the authors.

The paper is organized as follows.

In Section 2 we introduce some notation and define the general linear stochastic functional differential equation, which is used in Section 3 in the definitions of different kinds of stochastic stability. In this section we also offer a precise definition of $M$-stability for different spaces of stochastic processes. The central result of Section 3 describes relationship between stochastic Lyapunov stability and $M$ stability, where we relate specially defined spaces of stochastic processes to different kinds of stochastic Lyapunov stability.

Let us remark that the role of the definitions and results presented in Section 3 goes far beyond the applications to the stability analysis of the generalized stochastic pantograph equation. Bearing in mind these future applications, we chose to formulate and prove the results of Section 3 for the case of the general functional differential equation (2). In addition to (21), (2) covers integrodifferential equations arising, e.g., in electrical circuit analysis [18].

Section 4 contains applications to the stability analysis of the generalized stochastic pantograph equation (21), but we stress that most results are also new for the stochastic pantograph equation (1). This includes, e.g., conditions of $p$ stability for $p \neq 2$, stability with probability 1 , and stability conditions in the vector case.
Finally, Section 5 contains a short overview of the main results of the paper as well as some suggestions on further applications of the developed method.

\section{Notation and Preliminaries}

Let $\left(\Omega, \mathscr{F},\left(\mathscr{F}_{t}\right)_{t \geq 0}, P\right)$ be a stochastic basis (see, e.g., [19]), where $\Omega$ is a set of elementary probability events, $\mathscr{F}$ is a $\sigma$ algebra of all events on $\Omega,\left(\mathscr{F}_{t}\right)_{t>0}$ is a right continuous family of $\sigma$-subalgebras of $\mathscr{F}$, and $P$ is a probability measure on $\mathscr{F}$; all the above $\sigma$-algebras are assumed to be complete with respect to (w.r.t. in what follows) the measure $P$, i.e., they contain all subsets of zero measure; the symbol $E$ stands for the expectation related to the probability measure $P$.

In the sequel, we use an arbitrary yet fixed norm $|$.$| in R^{n}$, the real-valued index $p$ satisfying the assumption $0 \leq p \leq \infty$, and a continuous positive function $\gamma(t)$ defined for all $t \geq 0$.

By $Z=\left(z_{1}, \ldots, z_{m}\right)^{T}$ we denote an $m$-dimensional semimartingale (see, e.g., [19]), while $\mathscr{B}=\left(\mathscr{B}_{1}, \ldots, \mathscr{B}_{m}\right)^{T}$ stands for the standard Brownian motion (the Wiener process), the particular case of $Z$.

The general linear stochastic functional differential equation is defined as follows (see, e.g., [16]):

$$
d x(t)=(V x)(t) d Z(t) \quad(t \geq 0),
$$

and the initial condition reads in this case as

$$
x(0)=x_{0} \in R^{n} .
$$

Here $V$ is a $k$-linear Volterra operator (see below), which is defined in certain linear spaces of vector-valued stochastic processes.

By the $k$-linearity of the operator $V$ we mean the property

$$
V\left(\alpha_{1} x_{1}+\alpha_{2} x_{2}\right)=\alpha_{1} V x_{1}+\alpha_{2} V x_{2},
$$

which holds for all $\mathscr{F}_{0}$-measurable, bounded, and scalar random values $\alpha_{1}, \alpha_{2}$ and all stochastic processes $x_{1}, x_{2}$ belonging to the domain of the operator $V$.

According to [17] the following classes of linear stochastic equations can be represented as (2):

(a) Systems of linear ordinary (i.e., nondelay) stochastic differential equations driven by an arbitrary semimartingale (in particular, systems of ordinary Itô equations).

(b) Systems of linear stochastic differential equations with discrete delays driven by a semimartingale (in particular, systems of Itô equations with discrete delays).

(c) Systems of linear stochastic differential equations with distributed delays driven by a semimartingale (in particular, systems of Itô equations with distributed delays).

(d) Systems of linear stochastic integrodifferential equations driven by a semimartingale (in particular, systems of Itô integrodifferential equations).

(e) Systems of linear stochastic functional difference equations driven by a semimartingale (in particular, systems of Itô functional difference equations).

Of course, the stochastic pantograph equation (1) and its generalization (21) are also particular cases of the general functional differential equation (2). 


\section{Lyapunov Stability and $M$-Stability}

In this section we study different kinds of stochastic Lyapunov stability of the zero solution of the linear equation (2) with respect to the initial data (3). Let us start with the precise definitions.

Definition 1. The zero solution of (2) is called

(1) weakly stable in probability if for any $\epsilon>0, \delta>0$ there is $\eta(\epsilon, \delta)>0$ such that $P\left\{\omega \in \Omega:\left|x\left(t, x_{0}\right)\right|>\right.$ $\epsilon\}<\delta$ for all $\left|x_{0}\right|<\eta$ and $t \geq 0$;

(2) asymptotically weakly stable in probability if it is weakly stable in probability and if, in addition, for any $\epsilon>0$ and all $x_{0} \in R^{n}$ one has $P\left\{\omega \in \Omega:\left|x\left(t, x_{0}\right)\right|>\right.$ $\epsilon\} \rightarrow 0$ as $t \rightarrow+\infty$;

(3) stable in probability if for any $\epsilon, \delta>0$ there is $\eta(\epsilon, \delta)>$ 0 such that $P\left\{\omega \in \Omega: \sup _{t \geq 0}\left|x\left(t, x_{0}\right)\right|>\epsilon\right\}<\delta$ for all $\left|x_{0}\right|<\eta$;

(4) asymptotically stable in probability if it is stable in probability and if, in addition, for any $\varepsilon>0$ and all $x_{0} \in R^{n}$ one has $P\left\{\omega \in \Omega:\left|x\left(t, x_{0}\right)\right|>\epsilon\right\} \rightarrow 0$ as $t \rightarrow+\infty$;

(5) $p$-stable if for any $\epsilon>0$ there is $\eta(\epsilon)>0$ such that $\left|x_{0}\right|<\eta$ implies $E\left|x\left(t, x_{0}\right)\right|^{p} \leq \epsilon$ for all $t \geq 0$;

(6) asymptotically $p$-stable if it is $p$-stable and, in addition, $\lim _{t \rightarrow+\infty} E\left|x\left(t, x_{0}\right)\right|^{p}=0$ for all $x_{0} \in R^{n}$;

(7) exponentially $p$-stable if there exist positive constants $K, \beta$ such that the inequality $E\left|x\left(t, x_{0}\right)\right|^{p} \leq$ $K\left|x_{0}\right|^{p} \exp \{-\beta t\}$ holds true for all $t \geq 0$ and all $x_{0} \in$ $R^{n}$

(8) stable with probability 1 if $\sup _{t \geq 0}\left|x\left(t, x_{v}\right)\right| \rightarrow 0$ with probability 1 whenever $\left|x_{\nu}\right| \rightarrow 0$ as $\nu \rightarrow+\infty$;

(9) asymptotically stable with probability 1 if it is stable with probability 1 and if, in addition, $\left|x\left(t, x_{0}\right)\right| \rightarrow 0$ as $t \rightarrow+\infty$ for all $x_{0} \in R^{n}$

(10) strongly stable with probability 1 if for any $\epsilon>0$ there exists $\eta(\epsilon)>0$ such that $P\left\{\omega \in \Omega: \sup _{t \geq 0}\left|x\left(t, x_{0}\right)\right| \leq\right.$ $\epsilon\}=1$ whenever $\left|x_{0}\right|<\eta$;

(11) strongly asymptotically stable with probability 1 if it is strongly stable with probability 1 and if, in addition, for any $\epsilon>0 x\left(t, x_{0}\right)$ it tends to 0 with probability 1 as $t \rightarrow+\infty$ for all $x_{0} \in R^{n}$.

Remark 2. The initial condition $x_{0}$ can also be random. In this case the norm of $x_{0}$ should be adjusted accordingly.

For brevity, we will also write " (2) is stable" in a certain sense instead of "the zero solution of (2) is stable" in this sense.

In the sequel the following linear spaces of stochastic processes will be used:

(i) $L^{n}(Z)$ consists of all predictable $n \times m$-matrix stochastic processes on $[0,+\infty)$, the rows of which are locally integrable w.r.t. the semimartingale $Z$ (see, e.g., [19]).

(ii) $D^{n}$ consists of all $n$-dimensional stochastic processes on $[0,+\infty)$, which can be represented as

$$
x(t)=x(0)+\int_{0}^{t} H(s) d Z(s),
$$

where $x(0) \in R^{n}, H \in L^{n}(Z)$.

The spaces below (" $M$-spaces") are crucial for studying the stochastic Lyapunov stabilities listed above.

$M_{0}^{\gamma}=\left\{x: x \in D^{n}\right.$ such that for any $\delta>0$ there is $K>0$, for which $\left.\sup _{t \geq 0} P\{\omega: \omega \in \Omega,|\gamma(t) x(t)|>K\}<\delta\right\}$.

$\widehat{M}_{0}^{\gamma}=\left\{x: x \in D^{n}\right.$ such that for any $\delta>0$ there is $K>0$, for which $P\left\{\omega: \omega \in \Omega\right.$, $\left.\left.\sup _{t \geq 0}|\gamma(t) x(t)|>K\right\}<\delta\right\}$. $\infty)$.

$M_{p}^{\gamma}=\left\{x: x \in D^{n}, \sup _{t \geq 0} E|\gamma(t) x(t)|^{p}<\infty\right\}(0<p<$

$\widehat{M}_{p}^{\gamma}=\left\{x: x \in D^{n}, E \sup _{t \geq 0}|\gamma(t) x(t)|^{p}<\infty\right\}(0<p<$ $\infty)$. $\infty\}$.

$M_{\infty}^{\gamma}=\widehat{M}_{\infty}^{\gamma}=\left\{x: x \in D^{n}, \operatorname{ess}_{\sup }(t, \omega) \in[0,+\infty[\times \Omega|\gamma(t) x(t)|<\right.$

For $\gamma(t)=1(t \geq 0)$ we also put $M_{p}^{1}=M_{p}$ and $\widehat{M}_{p}^{1}=\widehat{M}_{p}$ $(0 \leq p \leq \infty)$.

Let $B$ be a linear subspace of the space $L^{n}(Z)$ equipped with some norm $\|\cdot\|_{B}$. For a given positive and continuous function $\gamma(t)(t \in[0, \infty))$ we define $B^{\gamma}=\{f: f \in B, \gamma f \in B\}$. The latter space becomes a linear normed space if we put $\|f\|_{B^{\gamma}}:=\|\gamma f\|_{B}$. By this, the linear spaces $M_{p}^{\gamma}, \widehat{M}_{p}^{\gamma}$ become normed spaces if $1 \leq p \leq \infty$.

Remark 3. The above spaces can also be described as follows. Let $L_{\infty}(X)$ be the space consisting of all essentially bounded functions $g:[0, \infty) \rightarrow X$, while $\mathscr{L}_{p}(Y)$ is the space of measurable $(p=0)$, $p$-integrable $(0<p<\infty)$, essentially bounded $(p=\infty)$ functions $h: \Omega \rightarrow Y$, where $X$ and $Y$ are arbitrary separable Banach spaces. Then it is easy to see that $M_{p}^{\gamma}=L_{\infty}\left(\mathscr{L}_{p}\left(R^{n}\right)\right)$ and $\widehat{M}_{p}^{\gamma}=\mathscr{L}_{p}\left(L_{\infty}\left(R^{n}\right)\right)$ for all $0 \leq p \leq \infty$ and an arbitrary positive and continuous function $\gamma:[0, \infty) \rightarrow R$. This means that the above list of the $M$ spaces covers all possible combinations of Lebesgue spaces with respect to the variable $\omega \in \Omega$ and spaces of essentially bounded functions with respect to the variable $t \in[0, \infty)$. As we will see, this list covers also all types of stochastic Lyapunov stability described in Definition 1.

Below we use the following assumptions on a continuous positive function $\gamma(t), t \in[0, \infty)$.

Property $\gamma 1$ : the function $\gamma$ satisfies the conditions $\gamma(t) \geq$ $\sigma(t \in[0,+\infty)), \sigma>0$, and $\lim _{t \rightarrow+\infty} \gamma(t)=+\infty$.

Property $\gamma 2: \gamma(t)=\exp \{\beta t\}$ for some $\beta>0$.

Definition 4. Let $x\left(., x_{0}\right)$ be the solution of the initial value problem (2)-(3) defined on $[0, \infty)$ and $\delta$ be a certain subspace of the space $D^{n}$. We say that (2) is $\mathcal{\delta}$-stable if $x\left(., x_{0}\right) \in \mathcal{S}$ for any $x_{0} \in R^{n}$.

Our first theorem describes relationships between the different kinds of the stochastic Lyapunov stability and the associated $\mathcal{S}$-stabilities for (2).

Theorem 5. The following statements describe relationship between stochastic stability properties of (2) and the M-spaces: 
(1) weak stability in probability is equivalent to the $M_{0^{-}}$ stability;

(2) weak asymptotic stability in probability is equivalent to the $M_{0}^{\gamma}$-stability for some $\gamma$ satisfying Property $\gamma 1$;

(3) stability in probability is equivalent to the $\widehat{M}_{0}$-stability;

(4) if $0<p<\infty$, then $p$-stability is equivalent to the $M_{p^{-}}$ stability;

(5) if $0<p<\infty$, then asymptotic $p$-stability is equivalent to the $M_{p}^{\gamma}$-stability for some $\gamma$ satisfying Property $\gamma 1$;

(6) if $0<p<\infty$, then exponential p-stability is equivalent to the $M_{p}^{\gamma}$-stability for some $\gamma$ satisfying Property $\gamma 2$;

(7) stability with probability 1 is equivalent to the $\widehat{M}_{0}$ stability;

(8) strong stability with probability 1 is equivalent to the $M_{\infty}$-stability.

Proof. We consider all cases separately.

$(1) \Leftarrow)$. Choosing arbitrary positive numbers $\epsilon$ and $\delta$ and minding that $x\left(t, x_{0}\right)=X(t) x_{0}$ for all $x_{0} \in R^{n}$ and all $t \geq 0$, we first find $K(\delta)>0$ such that $\sup _{t \geq 0} P\{\omega \in \Omega:\|X(t)\|>$ $K(\delta)\}<\delta$. Letting $\eta=\epsilon / K(\delta)$ we observe that $\left|x_{0}\right|<\eta$ implies $\sup _{t \geq 0} P\left\{\omega \in \Omega:\left|x\left(t, x_{0}\right)\right|>\epsilon\right\}<\delta$ for all $x_{0}$, $\left|x_{0}\right|<\eta$, which is equivalent to weak stability in probability.

$\Rightarrow$ ). Assume that the zero solution of (2) is weakly stable in probability. Using the notation from the previous part of the proof, we assume, in addition, that there exists $x_{0} \in R^{n}$ such that $x\left(., x_{0}\right)$ does not belong to $M_{0}$. Hence there is $\delta_{0}>0$ such that for any $\epsilon>0$

$$
P\left\{\omega \in \Omega:\left|x\left(t(\epsilon), x_{0}\right)\right|>\epsilon\right\}>\delta_{0}
$$

for at least one $t(\epsilon)>0$. In such a case, the solution $\widehat{x}(t) \equiv$ $\eta^{\prime} x\left(t, x_{0}\right) /\left|x_{0}\right|$, where $\left.0<\eta^{\prime}<\eta\right)$ satisfies the estimate $P\{\omega \in$ $\Omega:|x(t(\epsilon))|>\epsilon\}>\delta_{0}$, although $\left|x_{0}\right|<\eta$, which contradicts the assumption.

$(2) \Leftarrow)$. The weak stability in probability follows from the observation that $M_{0}^{\gamma}$ is topologically imbedded into $M_{0}$. Let us now take arbitrary $x_{0} \in R^{n}, \epsilon>0$, and $\delta>0$. As $x\left(., x_{0}\right) \epsilon$ $M_{0}^{\gamma}$, there exists $K>0$, for which

$$
\sup _{t \geq 0} P\left\{\omega \in \Omega:\left|\gamma(t) x\left(t, x_{0}\right)\right|>K\right\}<\delta .
$$

Now, choosing $T>0$ satisfying $K \gamma^{-1}(t)<\epsilon$ for all $t \geq T$ we obtain

$$
\begin{aligned}
& \sup _{t \geq 0} P\left\{\omega \in \Omega:\left|x\left(t, x_{0}\right)\right|>\epsilon\right\} \\
& \quad \leq \sup _{t \geq 0} P\left\{\omega \in \Omega:\left|\gamma(t) x\left(t, x_{0}\right)\right|>K\right\}<\delta,
\end{aligned}
$$

which implies weak asymptotic stability.

$\Rightarrow)$. From the weak asymptotic stability in probability and the representation $x\left(t, x_{0}\right)=X(t) x_{0}$ for all $x_{0} \in R^{n}$, we deduce that the function $\bar{\gamma}(t)=E(\|X(t)\| \wedge 1) \rightarrow 0$ as $t \rightarrow \infty$, as $E(|x-y| \wedge 1)$ is the metric in the space $L_{0}$. Then the function $\gamma(t)=1 / \bar{\gamma}(t)$ satisfies Property $\gamma 1$ with $\sigma=1$. Now, we take an arbitrary $x_{0} \in R^{n},\left|x_{0}\right| \leq 1$, and define $K=2 C \delta^{-1}$ where
$C=\sup _{t \geq 0} \bar{\gamma}(t)$. Let also choose $T>0$ satisfying the estimate $P\{\omega \in \Omega:\|X(t)\|>1\}<\delta / 2$ for all $t \geq T$. Then we obtain

$$
\begin{aligned}
P\{\omega & \left.\in \Omega:\left|x\left(t, x_{0}\right)\right|>K\right\} \leq P\{\omega \in \Omega:\|X(t)\|>K\} \\
\leq & P\{\omega \in \Omega:\|X(t)\|>1\} \\
& +P\{\omega \in \Omega:\|X(t)\| \wedge 1>K\} \\
\leq & \frac{\delta}{2}+\frac{\delta}{2 C} E(\|X(t)\| \wedge 1)=\frac{\delta}{2}+\frac{\delta}{2 C} \bar{\gamma}(t)<\delta
\end{aligned}
$$

for all $t \geq T$. This yields $\sup _{t \geq T} P\{\omega: \omega \in \Omega,|\gamma(t) x(t)|>$ $K\}<\delta$, which is sufficient to conclude that $x\left(., x_{0}\right) \in M_{0}^{\gamma}$, as $x\left(., x_{0}\right)$ is continuous with probability 1 and hence bounded in probability on the interval $[0, T]$.

$(3) \Leftarrow)$. This proof is similar to the one for case $(1)$, where we only need to replace $\sup _{t \geq 0} P\{\omega \in \Omega:\|X(t)\|>K(\delta)\}$ and $\sup _{t \geq 0} P\left\{\omega \in \Omega:\left|x\left(t, x_{0}\right)\right|>\epsilon\right\}$ by $P\left\{\omega \in \Omega: \sup _{t \geq 0}\|X(t)\|>\right.$ $K(\delta)\}$ and $P\left\{\omega \in \Omega: \sup _{t \geq 0}\left|x\left(t, x_{0}\right)\right|>\epsilon\right\}$, respectively.

$\Rightarrow)$. Assume that there exists $x_{0} \in R^{n}$ such that $x\left(., x_{0}\right)$ does not belong to $\widehat{M}_{0}$. Hence there is $\delta_{0}>0$ such that for any natural number $v>0$

$$
P\left\{\omega \in \Omega: \sup _{t \geq 0}\left|x\left(t, x_{0}\right)\right|>v\right\}>\delta_{0} .
$$

Then the solutions $x_{v}(t)=x\left(t, x_{0} v^{-1}\right)$ satisfy $P\{\omega \in \Omega$ : $\left.\sup _{t \geq 0}\left|x_{v}(t)\right|>1\right\}>\delta_{0}$, although the sequence $x_{n u}(0)=$ $x_{0} v^{-1}$ tends to 0 . This contradicts the property of stability in probability.

$(4) \Leftarrow)$. Let $e_{k}, k=1, \ldots, n$, be the standard basis in $R^{n}$, the latter being equipped with the norm $|b|=$ $\sum_{k=1}^{n}\left|b_{k}\right|$ (all norms are equivalent). Due to $p$-stability we have $\sup _{t \geq 0} E\left|x\left(t, e_{k}\right)\right|^{p} \leq K$ for some $K>0$ and any $k=$ $1, \ldots, n$. Using the associated matrix norm $\left\|\left[a_{1}, \ldots, a_{n}\right]\right\|=$ $\max _{1 \leq k \leq n}\left|a_{k}\right|$, where $a_{k} \in R^{n}$, yields $\sup _{t \geq 0} E\|X(t)\|^{p} \leq K$. For any $\epsilon>0$ we now define $\eta=(\epsilon / K)^{1 / p}$ obtaining

$$
\sup _{t \geq 0} E\left|x\left(t, x_{0}\right)\right|^{p} \leq \sup _{t \geq 0} E\|X(t)\|^{p}\left|x_{0}\right|^{p} \leq K \eta^{p}=\epsilon
$$

for any $\left|x_{0}\right|<\eta$, which completes the proof of $p$-stability.

$\Rightarrow)$. Assume, on the contrary, that for some $x_{0} \in R^{n}$ the solution $x\left(., x_{0}\right)$ does not belong to the space $M_{p}$. Hence for any natural number $v$ there exists $t_{v}$ such that $E\left|x\left(t_{\gamma}\right)\right|^{p}>v^{p}$. As $x_{\nu}(0)=x_{0} \nu^{-1}$ can be arbitrarily small in the norm, while $E\left|x_{v}\left(t_{v}\right)\right|^{p}>1$, this contradicts the property of $p$-stability.

$(5) \Leftarrow)$. As the space $M_{p}^{\gamma}$ is imbedded in $M_{p}$, the $p$ stability is trivial. On the other hand, for any $x_{0} \in R^{n}$ there exists a positive number $K$ such that $\sup _{t \geq 0} E \gamma(t)\left|x\left(t, x_{0}\right)\right|^{p} \leq$ $K$. Therefore $\sup _{t \geq 0} E\left|x\left(t, x_{0}\right)\right|^{p} \leq \gamma^{-1}(t) \rightarrow 0$ as $t \rightarrow \infty$ by Property $\gamma 1$.

$\Rightarrow)$. Using the same norm as in part (5), we obtain $E\|X(t)\|^{p} \rightarrow 0$ as $t \rightarrow \infty$, so that $\sup _{t \geq T} E\|X(t)\|^{p}<1$ for some $T>0$. Choosing $\bar{\gamma}(t)=E\left(\|X(t)\|^{p} \wedge 1\right)$ and putting $\gamma(t)=1 / \bar{\gamma}(t)$ (which satisfies Property $\gamma 1$ with $\sigma=1$ ), we obtain 


$$
\begin{aligned}
& \sup _{t \geq T} E\left(\gamma(t)\left|x\left(t, x_{0}\right)\right|^{p}\right) \leq \sup _{t \geq T} E\left(\gamma(t)\|X(t)\|^{p}\right)\left|x_{0}\right|^{p} \\
& \quad=\left|x_{0}\right|^{p} .
\end{aligned}
$$

As $\sup _{0 \leq t \leq T} E\left(\gamma(t)\left|x\left(t, x_{0}\right)\right|^{p}\right)$ is bounded due to continuity of the solutions, we deduce that $\sup _{t \geq T} E\left(\gamma(t)\left|x\left(t, x_{0}\right)\right|^{p}\right)<\infty$, so that $x\left(., x_{0}\right) \in M_{p}^{\gamma}$ for any $x_{0} \in R^{n}$.

$(6) \Leftarrow)$. Assume that $\gamma(t)=\exp \{\beta t\}$. Using the norm from (5), we conclude that $\sup _{t \geq 0} \gamma(t) E\left|x\left(t, e_{k}\right)\right|^{p} \leq K$ for all standard basis vectors $e_{k}$. This yields $\sup _{t \geq 0} \gamma(t) E\|X(t)\|^{p} \leq K$ and thus

$$
\begin{aligned}
E\left|x\left(t, x_{0}\right)\right|^{p} & \left.\leq E\|X(t)\|^{p} \mid x_{0}\right)\left.\right|^{p} \\
& \leq K\left|x_{0}\right|^{p} \exp \{-\beta t\} .
\end{aligned}
$$

$\Rightarrow)$. If $E\left|x\left(t, x_{0}\right)\right|^{p} \leq K\left|x_{0}\right|^{p} \exp \{-\beta t\}$ for all $t>0$ and $x_{0} \in R^{n}$, then $\sup _{t \geq 0} \gamma(t) E\left|x\left(t, x_{0}\right)\right|^{p} \leq K\left|x_{0}\right|^{p}$ is finite, so that $x\left(., x_{0}\right) \in M_{p}^{\gamma}$ for any $x_{0} \in R^{n}$.

$(7) \Leftarrow)$. We use again the norm from (5). According to the assumptions, for any $\delta>0$ there is $K=K(\delta)>0$, for which $P\left\{\omega \in \Omega: \sup _{t \geq 0}\left|x\left(t, e_{k}\right)\right|>K\right\}<\delta / n$ for all standard basis vectors $e_{k}$. Let $\Omega_{k}(\delta)$ contain all $\omega \in \Omega$ where $\sup _{t>0}\left|x\left(t, e_{k}\right)\right| \leq K$ and $\Omega(\delta)=\bigcap_{1 \leq k \leq n} \Omega_{k}(\delta)$. Evidently, $P \Omega(\delta) \geq 1-\delta$. As $\|X(t)\|=\left|x\left(t, e_{k}\right)\right|$ for some $e_{k}$ (dependent on $t$ and $\omega$ ), we have that $\sup _{t \geq 0}\|X(t)\| \leq K(\delta)$ for all $\omega \epsilon$ $\Omega(\delta)$. This means that $\sup _{t \geq 0}\|X(t)\|$ is almost surely finite.

Taking now an arbitrary sequence $x_{v} \in R^{n}$ going to 0 as $v \rightarrow \infty$, we conclude that

$$
\sup _{t \geq 0}\left|x\left(t, x_{v}\right)\right| \leq \sup _{t \geq 0}\|X(t)\|\left|x_{\nu}\right|
$$

tends to 0 almost surely. This completes the proof of this part.

$\Rightarrow)$. Given $x_{0} \in R^{n}$, the stability with probability 1 implies that the sequence $\sup _{t \geq 0} \mid x\left(t, x_{0} n^{-1}\right)$ converges to zero almost surely as $n \rightarrow \infty$. Thus, this sequence converges in probability, and therefore, for any $\delta>0$ there exists a number $N=N(\delta)$ such that $P\left\{\omega \in \Omega: \sup _{t \geq 0} \mid x\left(t, x_{0} / n\right) \geq 1\right\}<\delta$ for all $n \geq N$. Choosing $K(\delta)=N(\delta)$ we see that $P\{\omega \in \Omega$ : $\left.\sup _{t \geq 0} \mid x\left(t, x_{0}\right) \geq K\right\}<\delta$, so that $x\left(., x_{0}\right) \in \widehat{M}_{0}$.

$(8) \Leftarrow)$. Utilizing the norm from part (5) and the definition of the space $M_{\infty}$, we can write that, for some sufficiently large $K$, the estimates $\left.\sup _{t \geq 0}\left|x\left(t, e_{k}\right)\right| \leq K\right\}$ hold for all standard basis vectors $e_{k}, k=1, \ldots, n$, and all $\omega \in \Omega_{k}$, where $P \Omega_{k}=1$. Therefore, on the set $\widehat{\Omega}=\bigcap_{1 \leq k \leq n} \Omega_{k}$ of full measure we have

$$
\begin{aligned}
\sup _{t \geq 0}\|X(t)\| & \leq \sup _{t \geq 0} \max _{1 \leq k \leq n}\left|x\left(t, e_{k}\right)\right|=\operatorname{maxsup}_{1 \leq k \leq n}\left|x\left(t, e_{k}\right)\right| \\
& \leq K .
\end{aligned}
$$

Hence, given $\delta>0$ we set $\eta=\delta / K$ and obtain

$$
\begin{aligned}
& P\left\{\omega: \omega \in \Omega, \sup _{t \geq 0}\left|x\left(t, x_{0}\right)\right|\right\} \\
& \quad \leq P\left\{\omega: \omega \in \Omega, \sup _{t \geq 0}\|X(t)\|\left|x_{0}\right|\right\} \leq \delta
\end{aligned}
$$

as long as $x_{0} \in R^{n},\left|x_{0}\right|<\eta$. By this, we verified strong stability with probability 1 .
$\Rightarrow)$. Assume that there exists a solution $x\left(., x_{0}\right)$ that does not belong to the space $M_{\infty}$. This means that there exists $\delta_{0}>0$ such that for any natural number $\nu P\{\omega: \omega \epsilon$ $\left.\Omega, \sup _{t \geq 0}\left|x\left(t, x_{0}\right)\right| \leq \nu\right\} \leq 1-\epsilon_{0}$. Defining $x_{\nu}(t)=x\left(t, x_{0} v^{-1}\right)$ we see that $P\left\{\omega: \omega \in \Omega\right.$, $\left.\sup _{t \geq 0}\left|x_{n u}(t)\right| \leq 1\right\} \leq 1-\epsilon_{0}$, although $x_{v}(0)=x_{0} v^{-1}$ goes to 0 as $v \rightarrow \infty$. This contradicts the property of strong stability with probability 1 .

Remark 6. Note that the $M$-stabilities listed in Theorem 5 imply the respective stability properties not only w.r.t. the initial values $x_{0} \in R^{n}$, but also w.r.t. the random initial values, provided that the increments of the semimartingale $Z$ are independent of the initial $\sigma$-algebra $\mathscr{F}_{0}$. This is, e.g., the case if the family $\left(\mathscr{F}_{t}\right)_{t \geq 0}$ is generated by the Brownian motion. This comment applies to all results proven below.

Lemma 7. Let $p, q \in[0, \infty]$ and $\gamma, \kappa$ be continuous functions defined on $[0, \infty)$ and satisfying Property $\gamma 1$. Then the following inclusions are topological embedding:

(1) $M_{p}^{\gamma} \subset M_{q}^{\gamma}$ and $\widehat{M}_{p}^{\gamma} \subset \widehat{M}_{q}^{\gamma}$ if $p \geq q$;

(2) $M_{p}^{\gamma} \subset M_{p}^{\kappa}$ and $\widehat{M}_{p}^{\gamma} \subset \widehat{M}_{p}^{\kappa}$ if $\gamma(t) \geq \kappa(t)$ for $t \geq t_{0}$ $\left(t_{0} \geq 0\right.$ is some real number);

(3) $\widehat{M}_{p}^{\gamma} \subset M_{p}^{\gamma}$.

Proof. The first statement simply follows from the standard relationship between Lebesgue spaces generated by finite measure sets. The second and the third statements are a direct consequence of the definitions of the spaces $M_{p}^{\gamma}$ and $\widehat{M}_{p}^{\gamma}$ and the inequality $\sup _{t \geq 0} E|y(t)| \leq E \sup _{t \geq 0}|y(t)|$, which holds for any measurable stochastic process $y(t)$.

Theorem 8. The following is valid for the stochastic functional differential equation (2):

(1) if $0<q<p<\infty$, then $p$-stability (resp. asymptotic, exponential p-stability) implies q-stability (resp., asymptotic, exponential q-stability);

(2) if $0<p<\infty$, then $p$-stability (resp., asymptotic p-stability) implies weak stability in probability (resp., weak asymptotic stability in probability);

(3) stability in probability (resp., asymptotic stability in probability) implies weak stability with probability 1 (resp., weak asymptotic stability with probability 1).

(4) stability in probability is equivalent to stability with probability 1 .

Proof. The first and the second part follow directly from the first statement of Lemma 7 combined with statements (4)(6) and (1)-(2) and (4)-(5) of Theorem 5, respectively. The third part follows from the second statement of Lemma 7 combined with statements (1)-(4) of Theorem 5. To prove the last part first we observe that statements (3) and (8) of Theorem 5 contain the same space $M_{0}^{\gamma}$, so that stability in probability and stability with probability 1 are equivalent for (2).

In the next theorem, we describe more relations between different kinds of the stochastic Lyapunov stability. Some of these results are used in the examples below. 
Theorem 9. Let $p \in[0, \infty]$. Then

(1) $\widehat{M}_{p}$-stability implies stability with probability 1.

(2) $\widehat{M}_{p}^{\gamma}$-stability with $\gamma$ satisfying Property $\gamma 1$ implies asymptotic stability with probability 1.

(3) $\widehat{M}_{\infty}^{\gamma}$-stability with $\gamma$ satisfying Property $\gamma 1$ implies strong asymptotic stability with probability 1.

Proof. (1). This follows from statement (7) of Theorem 5 and the inclusion $\widehat{M}_{p} \subset \widehat{M}_{0}$.

(2). Due to the first statement of the theorem and the inclusion $\widehat{M}_{p}^{\gamma} \subset \widehat{M}_{0}^{\gamma}$ we obtain the property of stability with probability 1 . To prove asymptotic stability with probability 1 , we proceed as in the proof of statement (7) of Theorem 5 adding $\gamma(t)$ to $x\left(t, x_{0}\right)$ and $X(t)$, which gives almost everywhere boundedness of the random function $\xi=\xi(\omega)=$ $\sup _{t \geq 0} \gamma(t)\|X(t)\|$. Therefore

$$
\gamma(t)\left|x\left(t, x_{0}\right)\right| \leq \sup _{t \geq 0} \gamma(t)\|X(t)\|\left|x_{0}\right|=\xi\left|x_{0}\right|,
$$

so that

$$
\left|x\left(t, x_{0}\right)\right| \leq \frac{\xi\left|x_{0}\right|}{\gamma(t)} \longrightarrow 0
$$

$$
\text { almost everywhere as } t \longrightarrow \infty \text {. }
$$

(3). As $\widehat{M}_{\infty}^{\gamma} \subset \widehat{M}_{\infty}$, we readily obtain strong asymptotic stability with probability 1 from the last statement of Theorem 5. Adding $\gamma(t)$ to $x\left(t, x_{0}\right)$ and $X(t)$ and following the proof of this statement yield the estimate $\sup _{t \geq 0} \gamma(t)\|X(t)\| \leq$ $K$ for some (nonrandom) constant $K$. Hence

$$
\gamma(t)\left|x\left(t, x_{0}\right)\right| \leq \sup _{t \geq 0} \gamma(t)\|X(t)\|\left|x_{0}\right| \leq K\left|x_{0}\right|,
$$

and $\left|x\left(t, x_{0}\right)\right| \leq K\left|x_{0}\right| / \gamma(t) \rightarrow 0$ with probability 1 as $t \rightarrow$ $\infty$.

\section{Delay-Independent Stability Conditions for the Generalized Pantograph Equation Driven by the Brownian Motion}

Delay-independent stability is also called absolute stability (see, e.g., [20]). This property is important if the delay functions are unknown or difficult to determine.

It is well-known (see, e.g., [3]) that using Itô's formula usually results in a delay-dependent stability condition for stochastic pantograph equations. Below we present an alternative method which is based on the theory developed in Section 3.

We start with the analysis of $p$-stability, where we use the integral form of the Marcinkiewicz-Zygmund inequality

$$
E\left|\int_{0}^{t} \zeta(s) d \mathscr{B}(s)\right|^{2 p} \leq \rho_{p}^{2 p} E\left(\int_{0}^{t}|\zeta(s)|^{2} d s\right)^{p},
$$

which holds true for any predictable stochastic process $\zeta(s)$ $(0 \leq s \leq t)$, any $t>0$, and the scalar standard Brownian motion $\mathscr{B}(s)$. The constant $\rho_{p}$ depends on the number $p$ $(0<p<\infty)$, only. In 1988 D.L. Burkholder proved (see, for example, $[21,22])$ that the constant $\rho_{p}=2 p-1$ in the Marcinkiewicz-Zygmund inequality (20) is best possible for all for $p \geq 1$.

Consider the following equation:

$$
\begin{aligned}
d x(t)= & {\left[A x(t)+\sum_{j=0}^{m_{0}} A_{j 0} x\left(h_{j 0}(t)\right)\right] d t } \\
& +\sum_{l=1}^{m-1} \sum_{j=0}^{m_{l}} A_{j l} x\left(h_{j l}(t)\right) d \mathscr{B}_{l}(t) \quad(t \geq 0) .
\end{aligned}
$$

For all $0 \leq l \leq m-1,0 \leq j \leq m_{l}$, the $n \times n$-matrices $A, A_{j l}$ contain constant, real entries, the standard Wiener processes $B_{l}(t)$ are independent, and the functions $h_{j l}$ are Borel measurable functions satisfying $0 \leq h_{j l}(t) \leq t$ on $[0, \infty)$.

In the next theorem we put $A_{l}=\sum_{j=0}^{m_{l}}\left\|A_{j l}\right\|$ for all $l=$ $0, \ldots, m-1$, where $\|$.$\| is the matrix norm induced by the$ chosen vector norm |.|.

Theorem 10. Let $p$ satisfy the inequality $1 \leq p<\infty$. Assume that there exists a positive number $\alpha$ such that

$$
\left\|A+\alpha I_{n}\right\|+A_{0}+\rho_{p} \sqrt{\frac{\alpha}{2}} \sum_{l=1}^{m-1} A_{l}<\alpha,
$$

where $I_{n}$ is the $n \times n$ identity matrix. Then (21) is $2 p$-stable.

Proof. We apply the forth statement from Theorem 5. To show that the solutions $x(t)$ of (21) belong to the space $M_{2 p}$ for all $x(0) \in R^{n}$, we replace this equation by an equivalent integral equation

$$
\begin{aligned}
x(t) & =e^{-\alpha t} x(0)+\int_{0}^{t} e^{-\alpha(t-s)}\left[\left(A+\alpha I_{n}\right) x(s)\right. \\
& \left.+\sum_{j=0}^{m_{0}} A_{j 0} x\left(h_{j 0}(s)\right)\right] d s \\
& +\sum_{l=1}^{m-1} \sum_{j=0}^{m_{l}} \int_{0}^{t} e^{-\alpha(t-s)} A_{j l} x\left(h_{j l}(s)\right) d \mathscr{B}_{l}(s)
\end{aligned}
$$

$(t \geq 0)$.

Using the Marcinkiewicz-Zygmund inequality (20) we obtain

$$
\begin{aligned}
& \left(\sup _{t \geq 0} E|x(t)|^{2 p}\right)^{1 / 2 p} \leq|x(0)|+\left(\sup _{t \geq 0} E\right. \\
& \left.\cdot\left|\int_{0}^{t} e^{-\alpha(t-s)}\left(A+\alpha I_{n}\right) x(s) d s\right|^{2 p}\right)^{1 / 2 p} \\
& +\sum_{j=0}^{m_{0}}\left(\sup _{t \geq 0} E\left|\int_{0}^{t} e^{-\alpha(t-s)} A_{j 0} x\left(h_{j 0}(s)\right) d s\right|^{2 p}\right)^{1 / 2 p}
\end{aligned}
$$




$$
\begin{aligned}
& +\sum_{l=1}^{m-1} \sum_{j=0}^{m_{l}}\left(\sup _{t \geq 0} E\right. \\
& \left.\cdot\left|\int_{0}^{t} e^{-\alpha(t-s)} A_{j l} x\left(h_{j l}(s)\right) d \mathscr{B}_{l}(s)\right|^{2 p}\right)^{1 / 2 p} \\
& \leq|x(0)|+\alpha^{-1}\left\|A+\alpha I_{n}\right\|\left(\sup _{t \geq 0} E|x(t)|^{2 p}\right)^{1 / 2 p} \\
& +\alpha^{-1} \sum_{j=0}^{j=m_{0}}\left\|A_{j 0}\right\|\left(\sup _{t \geq 0} E\left|x\left(h_{j 0}(t)\right)\right|^{2 p}\right)^{1 / 2 p} \\
& +\rho_{p} \sum_{l=1}^{m-1} \sum_{j=0}^{j=m_{l}}\left(\sup _{t \geq 0} E\right. \\
& \left.\cdot\left(\int_{0}^{t} e^{-2 \alpha(t-s)}\left\|A_{j l}\right\|^{2}\left|x\left(h_{j l}(s)\right)\right|^{2} d s\right)^{p}\right)^{1 / 2 p} \\
& \leq|x(0)|+K\left(\sup _{t \geq 0} E|x(t)|^{2 p}\right)^{1 / 2 p},
\end{aligned}
$$

where $K=\alpha^{-1}\left\|A+\alpha I_{n}\right\|+\alpha^{-1} A_{0}+\rho_{p}(2 \alpha)^{-1 / 2} \sum_{l=1}^{m-1} A_{l}$. In the estimates above we used the assumptions $0 \leq h_{j l}(t) \leq t$ for all $t \geq 0,0 \leq j \leq m_{l}, 0 \leq l \leq m-1$. Due to (22), the constant $K$ is less than 1 , so that

$$
\left(\sup _{t \geq 0} E|x(t)|^{2 p}\right)^{1 / 2 p} \leq(1-K)^{-1}|x(0)| .
$$

Therefore $x \in M_{2 p}$ for all $x(0) \in R^{n}$.

Consider the generalized scalar pantograph equation

$$
\begin{aligned}
d x= & {[a x(t)+b x(h(t)] d t} \\
& +[c x(t)+d x(g(t))] d \mathscr{B}(t) \quad(t \geq 0),
\end{aligned}
$$

where $a, b, c, d$ are real constants, $h(t)$ and $g(t)(t \geq 0)$ are Borel measurable functions satisfying $0 \leq h(t) \leq t, 0 \leq g(t) \leq$ $t(t \geq 0)$, and $\mathscr{B}(t)$ is the standard scalar Brownian motion.

Corollary 11. Let $\xi_{0}$ be the (only) positive root of the quadratic polynomial $P(\xi)=\xi^{2}-K \xi-|b|$, where $K=\rho_{p}((|c|+|d|) / \sqrt{2})$. If $a<-\xi_{0}^{2}$, then (26) is $2 p$-stable.

Proof. Inequality (22) reads in this case as follows:

$$
|a+\alpha|+|b|+K \sqrt{\alpha}<\alpha .
$$

Assuming that $a<-\xi_{0}^{2}$ and putting $\alpha=\xi_{0}^{2}>-a>0$ we observe that

$$
\alpha-|a+\alpha|-|b|-K \sqrt{\alpha}=\xi^{2}-|b|-K \xi>0,
$$

as $\xi>\sqrt{-a}>\xi_{0}$.
Let us compare Corollary 11 with the following wellknown result (see [1] or [2]): (26) with the linear delays $h(t)=$ $g(t)=k t(0<k<1)$ is 2-stable if $2 a+2|b+c d|+c^{2}+d^{2}<0$.

In the case when $b=0$, this result and Corollary 11 give the same condition: $a<-(|c|+|d|)^{2} / 2$.

If $c=0$, then the result from [1] gives, in fact, a better stability condition in the case of linear delays: $a<-d^{2} / 2-|b|$ versus $a<-d^{2} / 4-|b|-(d / \sqrt{2}) \sqrt{-d^{2} / 8+|b|}$, but our result covers more general delays and the case of $p$-stability for $p \neq$ 2.

Now we briefly describe explicit stability conditions for one particular case of the vector equation (21).

Corollary 12. Let the matrix $A$ have only real eigenvalues $\lambda_{i}(1 \leq i \leq n)$ and $A_{j l}$ be scalar matrices:

$$
\begin{aligned}
& A_{j l}=\operatorname{diag}\left[a_{j l}, \ldots, a_{j l}\right] \\
& \qquad\left(0 \leq l \leq m-1 ; 1 \leq j \leq m_{l}\right) .
\end{aligned}
$$

Then (21) is 2p-stable if $\lambda_{i}<-\xi_{0}^{2}(1 \leq i \leq n)$, where $\xi_{0}$ is the only positive root of the quadratic polynomial

$$
P_{i}(\xi)=\xi^{2}-K \xi-a_{0}
$$

and

$$
K=\frac{\rho_{p}}{\sqrt{2}} \sqrt{\sum_{l=1}^{m-1} \sum_{j=0}^{m_{l}}\left\|a_{j l}\right\|}
$$

Proof. Let us choose $\alpha=\xi_{0}^{2}$. To verify condition (22) of Theorem 10 we first observe that for any positive $\epsilon>0$ there exists a basis in $R^{n}$, in which the Euclidean matrix norm $\|$. satisfies $\left\|A+\alpha I_{n}\right\|<|\lambda+\alpha|+\epsilon$, where $\lambda$ is the largest eigenvalue of the matrix $A$. At the same time, the Euclidean norms of the scalar matrices $A_{j l}\left(0 \leq l \leq m-1 ; 1 \leq j \leq m_{l}\right)$ are independent of the choice of the basis. Denoting

$$
A_{l}=\sum_{j=0}^{m_{l}}\left\|a_{j l}\right\|
$$

for all $l=0, \ldots, m-1$ we obtain, exactly in the same manner as in the proof of Corollary 11, that

$$
\begin{aligned}
\| A & +\alpha I_{n} \|+A_{0}+\rho_{p} \sqrt{\frac{\alpha}{2}} \sum_{l=1}^{m-1} A_{l} \\
& <|\lambda+\alpha|+\epsilon+A_{0}+K \sqrt{\alpha}<\alpha
\end{aligned}
$$

for sufficiently small $\epsilon$.

In the next theorem, where we study stability with probability 1 , we use the Doob inequality

$$
E \sup _{t \geq 0}\left|\int_{0}^{t} \zeta(s) d \mathscr{B}(s)\right|^{2} \leq 4 E\left(\sup _{t \geq 0} \int_{0}^{t}|\zeta(s)|^{2} d s\right)
$$

where $\mathscr{B}$ is the scalar standard Brownian motion and $\zeta$ is an arbitrary predictable stochastic process on $[0, \infty)$. 
Theorem 13. Assume that there exists a positive number $\alpha$ such that

$$
\left\|A+\alpha I_{n}\right\|+A_{0}+\sqrt{2 \alpha} \sum_{l=1}^{m-1} A_{l}<\alpha,
$$

where $I_{n}$ is the $n \times n$ identity matrix. Then (21) is stable with probability 1 .

Proof. We want to apply the first statement from Theorem 9 by checking that the solutions $x(t)$ of (21) belong to the space $\widehat{M}_{2}$ for all $x(0) \in R^{n}$. This is done exactly in the same way as in the proof of Theorem 10 provided that the norm $\left(\sup _{t \geq 0} E|x(t)|^{2 p}\right)^{1 / 2 p}$ is replaced by the norm $\left(E \sup _{t \geq 0}|x(t)|^{2 p}\right)^{1 / 2 p}$ and the Doob inequality is used instead of the Marcinkiewicz-Zygmund inequality.

\section{Conclusions and Outlook}

In the paper we described and justified a new framework for stability analysis of stochastic functional differential equations in the case when initial data are finite dimensional. In particular, this framework covers the generalized stochastic pantograph equation. The main feature of our analysis consists in replacing Lyapunov stabilities with input-tostate stabilities by choosing appropriate spaces of stochastic processes. It is shown that this approach is applicable to all known kinds of stochastic Lyapunov stability.

We demonstrated the efficiency of this idea by applying it to the generalized stochastic pantograph equation, where the emphasis was put on delay-independent stability conditions. In particular, we studied $2 p$-stability $(p \geq 1)$ and stability with probability 1 .

The future development of the suggested framework may include the following topics:

(1) Stability analysis of the stochastic pantograph equations may be extended to the case of nonconstant coefficients. In this case, Theorem 5 and other results of Section 3 could still be applied.

(2) More attention should be paid to the vector case. In particular, coefficient-based stability conditions may be derived from Theorems 10 and 13 to get more general stability tests than those offered in Section 4 .

(3) Asymptotic $p$-stability and asymptotic stability with probability 1 are only described in terms of $M$ stability, but not in the form of specific stability conditions, so that further analysis of these asymptotic properties should be continued.

(4) The stochastic pantograph equation driven by an arbitrary semimartingale can also be studied using the techniques developed in Section 3.

(5) The main results of Section 3 stretch are far beyond the generalized pantograph equation. As it is mentioned in Section 2, wide classes of stochastic functional differential equations can be represented as (2). The technique developed in this paper can be, e.g., directly applied to integrodifferential equations, where

$$
\begin{gathered}
(V x)(t)=\left(\int_{(0, t)} d_{s} \mathscr{R}_{1}(t, s) x(s), \ldots,\right. \\
\left.\int_{(0, t)} d_{s} \mathscr{R}_{m}(t, s) x(s)\right),
\end{gathered}
$$

$\mathscr{R}_{i}$ are vector functions defined on $\{(t, s): t \in[0$, $\infty), 0<s \leq t\}$ for $i=1, \ldots, m$. But this analysis is beyond the scope of the present paper.

(6) Stochastic hereditary equations with more general initial conditions $x(s)=\varphi(s), s \leq 0$, can easily be transformed into (2) as well (see, e.g., [16]). However, a possible generalization of the main results of Section 3 will not be straightforward, as in this case, one will, in addition, need to prove boundedness of the infinite dimensional linear operators assigning $\varphi$ to the associated solution $x(t)$.

\section{Data Availability}

No data were used to support this study, which is purely theoretical.

\section{Conflicts of Interest}

The authors declare that they have no conflicts of interest.

\section{Acknowledgments}

The authors have been partially supported by the Norwegian Research Council, Grant no. 239070.

\section{References}

[1] Z.-c. Fan and M.-z. Liu, "The asymptotically mean square stability of the linear stochastic pantograph equation," Mathematica Applicata, vol. 20, no. 3, pp. 519-523, 2007.

[2] F. Xiao and P. Wang, "Strong predictor-corrector methods for stochastic pantograph equations," Journal of Computational Mathematics, vol. 34, no. 1, pp. 1-11, 2016.

[3] Y. Zhang and L. Li, "Stability of numerical method for semilinear stochastic pantograph differential equations," Journal of Inequalities and Applications, vol. 30, 2016.

[4] X. Feng, "An analytic study on the multi-pantograph delay equations with variable coefficients," Bull. Math. Soc. Sci. Math. Roumanie, vol. 56(104), no. 2, pp. 205-215, 2013.

[5] B. G. Grebenshchikov, "Methods for studying the stability of systems with linear delay," Siberian Math. J, vol. 42, no. 1, pp. 41-51, i, 2001.

[6] A. Iserles, "On the generalized pantograph functionaldifferential equation," European Journal of Applied Mathematics, vol. 4, no. 1, pp. 1-38, 1993.

[7] R. I. Kadiev, "Asymptotic stability of itô differential systems with retarded argument," Journal of Differential Equations, vol. 36, no. 2, pp. 187-192, 2000. 
[8] M. Z. Liu and D. Li, "Properties of analytic solution and numerical solution of multi-pantograph equation," Applied Mathematics and Computation, vol. 155, no. 3, pp. 853-871, 2004.

[9] X. Meng and B. Yin, "On the general decay stability of stochastic differential equations with unbounded delay," Journal of the Korean Mathematical Society, vol. 49, no. 3, pp. 515-536, 2012.

[10] Z. Lu, T. Yang, Y. Hu, and J. Hu, "Convergence rate of numerical solutions for nonlinear stochastic pantograph equations with Markovian switching and jumps," Abstract and Applied Analysis, 12 pages, 2015.

[11] H. Yang, F. Jiang, and Y. Jiang, "Robust stability of stochastic pantograph differential equations Markovian switching," in International Conference on Computer Application and System Modelling (ICCASM), vol. 5, pp. 541-545, Shanxi, Taiyuan, 2010.

[12] J. A. Appleby and E. Buckwar, "A constructive comparison technique for determining the asymptotic behaviour of linear functional differential equations with unbounded delay," Differential Equations and Dynamical Systems, vol. 18, no. 3, pp. 271301, 2010.

[13] J. A. D. Appleby and E. Buckwar, "Sufficient conditions for polynomial asymptotic behaviour of the stochastic pantograph equation," in Proceedings of the El. J. of Qual. Th. Diff. Equ., Proc. 10th Coll. Qualitative Theory of Diff. Equ., Electron. J. Qual. Theory Differ. Equ., Szeged, Szeged, Hungary, July 1-4, 2015.

[14] J. L. Massera and J. J. Schaeffer, Linear Differential Equations and Function Spaces, Elsevier, 1966.

[15] N. V. Azbelev and P. M. Simonov, Stability of Differential Equations with Aftereffect, Taylor and Francis, London, UK, 2002.

[16] R. Kadiev and A. Ponosov, "Input-to-state stability of linear stochastic functional differential equations," Journal of Function Spaces, Art. ID 8901563, 12 pages, 2016.

[17] R. I. Kadiev and A. V. Ponosov, "Stability of linear stochastic functional-differential equations with constantly acting perturbations," Diff. Eqs, vol. 28, no. 2, pp. 198-207, 364, 1992.

[18] V. Lakshmikantham and M. R. M. Rao, Theory of IntegroDifferential Equations, CRC Press, 1995.

[19] R. S. Liptser and A. N. Shiryayev, Theory of Martingales, Kluwer Academic Publishers, Dordrecht, The Netherlands, 1989.

[20] S. Ruan, "Absolute stability, conditional stability and bifurcation in Kolmogorov-type predator-prey systems with discrete delays," Quarterly of Applied Mathematics, vol. 59, no. 1, pp. 159173, 2001.

[21] D. Ferger, "Optimal constants in the Marcinkiewicz-Zygmund inequalities," Statistics \& Probability Letters, vol. 84, no. 1, pp. 96-101, 2014.

[22] Y. Ren and H. Liang, "On the best constant in MarcinkiewiczZygmund inequality," Stat. Probab. Letters, vol. 3, no. 15, pp. 227233, 2001. 


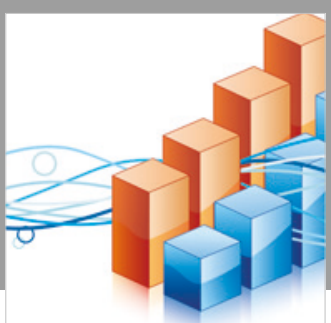

Advances in

Operations Research

\section{-n-m}
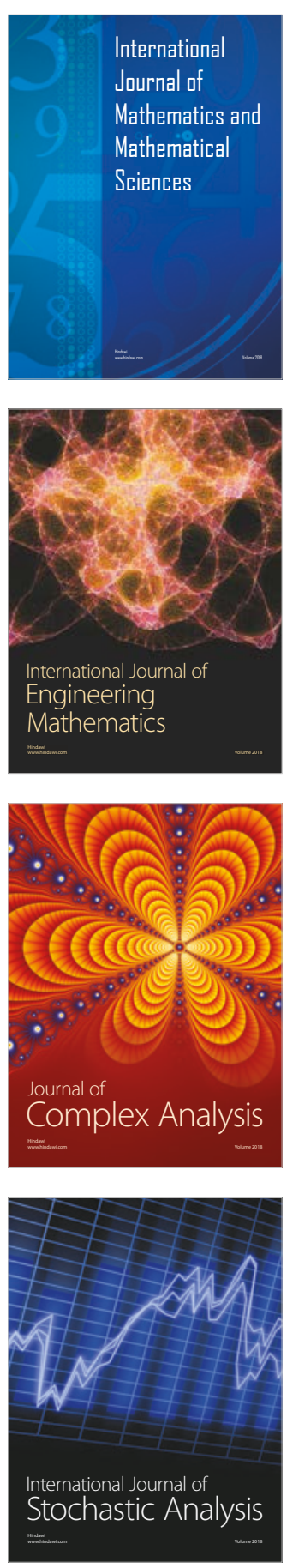
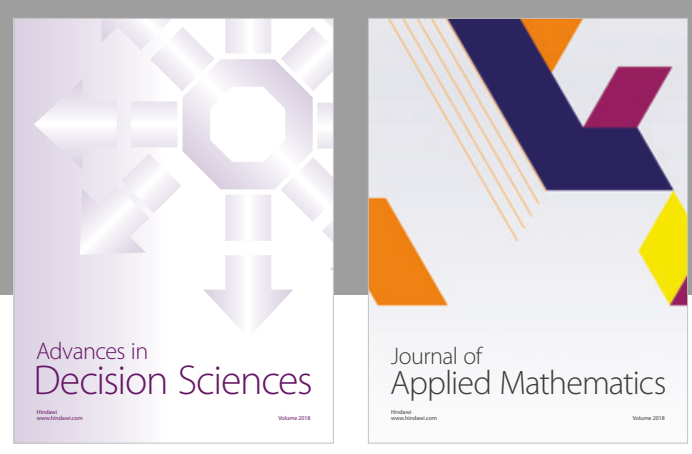

Journal of

Applied Mathematics
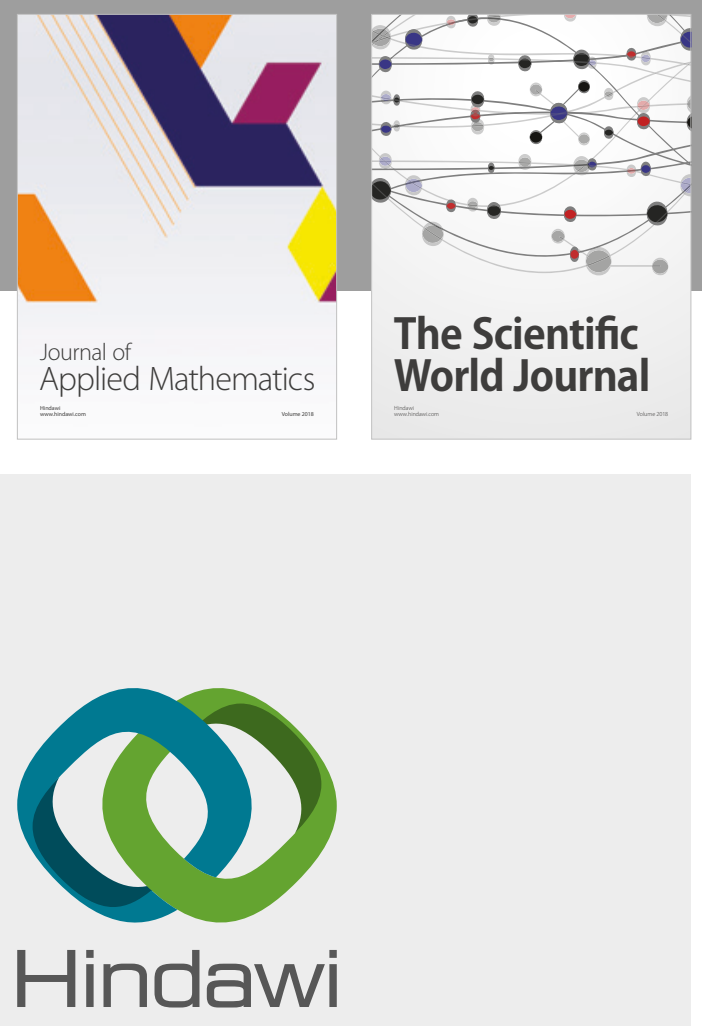

Submit your manuscripts at

www.hindawi.com

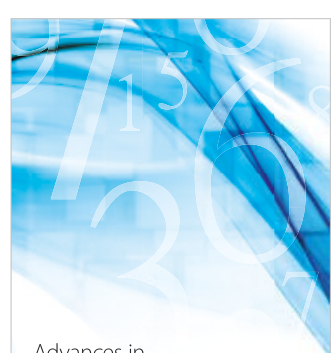

Advances in
Numerical Analysis
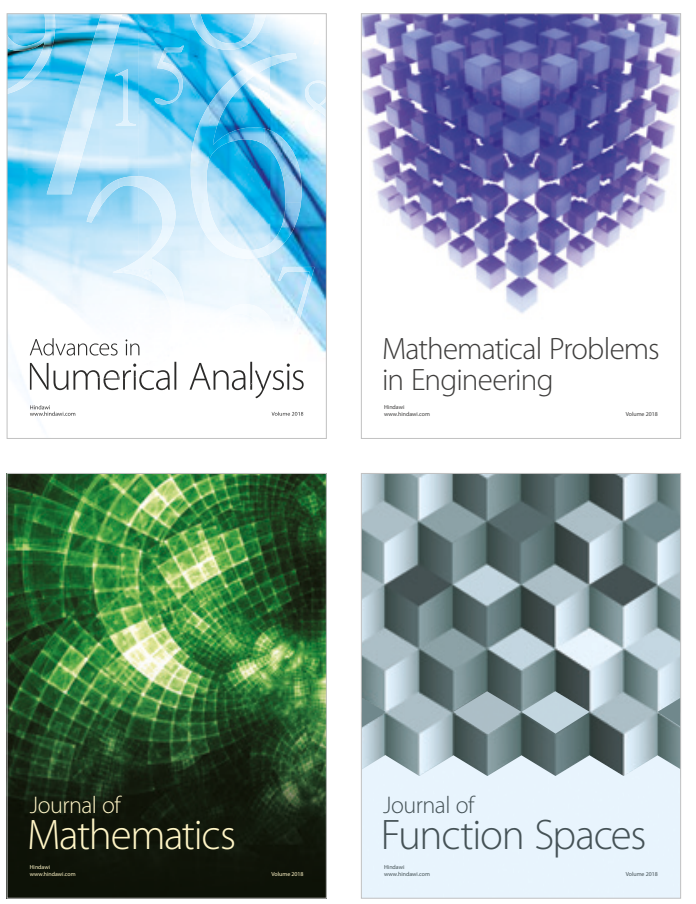

Mathematical Problems in Engineering

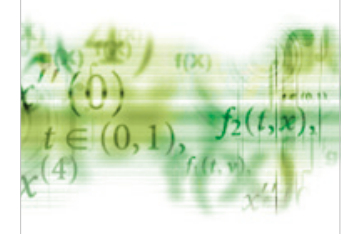

International Journal of

Differential Equations

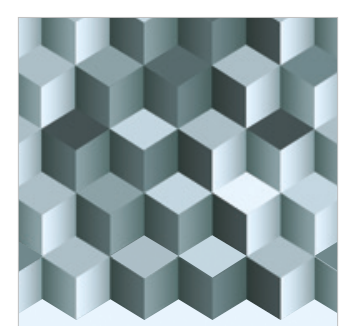

Journal of

Function Spaces
The Scientific

World Journal

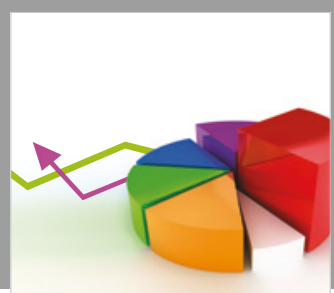

Journal of

Probability and Statistics
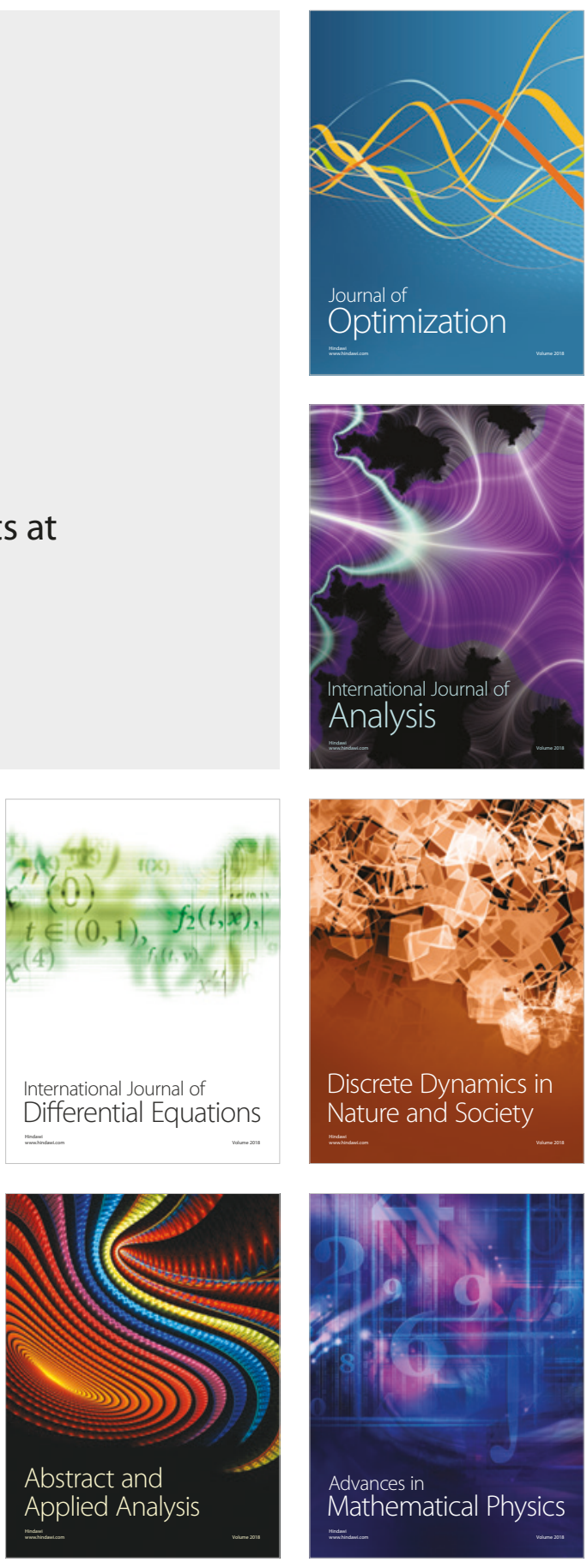